(2) Open Access Full Text Article

\title{
Anastomoses (Superficial Cervical Ansa) Between the Cervical Plexus and Peripheral Facial Nerve Branches: Implications for Regional Anesthesia in Carotid Endarterectomies - Anatomical Study
}

\author{
Ronald Seidel (D) \\ Andreas Wree ${ }^{2}$ \\ Marko Schulze (D) $^{3}$ \\ 'Asklepios Medical Center, Department \\ of Anesthesiology and Intensive Care, \\ Schwedt, 16303, Germany; ${ }^{2}$ Rostock \\ University Medical Center, Institute of \\ Anatomy, Rostock, DE-18057, Germany; \\ ${ }^{3}$ Bielefeld University Medical Center \\ OWL, Working Group 3: Anatomy and \\ Cell Biology, Bielefeld, DE-3350I, \\ Germany
}

\begin{abstract}
Purpose: Sensory innervation in the carotid triangle involves the cervical plexus, cranial nerves, and the sympathetic trunk. This innervation also applies to skin incision, including various anatomical structures with potentially different innervation, such as the skin (dermatomes), the platysma (myotomes), and the superficial layer of the cervical fascia (fasciotomes), as well as retromandibular retractor insertion (co-innervation: V, VII). The aim of this anatomical study was to develop an injection technique for carotid endarterectomies to additionally block anastomoses between the transverse cervical nerve (TCN), the cervical branch VII (CB VII), and the marginal mandibular branch VII (MMB VII). These anastomoses are also termed superficial cervical ansa (SCA).
\end{abstract}

Materials and Methods: Preparations $(n=16)$ were performed on unembalmed donor cadavers $(\mathrm{n}=8)$. Subplatysmal injections (each using $5 \mathrm{~mL}$ of Alcian blue) were performed cranially within the carotid triangle between the anterior margin of the sternocleidomastoid muscle and the submandibular gland.

Results: Anastomoses between the TCN, CB VII, and MMB VII were stained in all preparations $(\mathrm{n}=16)$.

Conclusion: This anatomical study presents an ultrasound-guided subplatysmal SCA block to optimize, in addition to a cervical plexus block, the quality of anesthesia for carotid endarterectomies.

Keywords: internal carotid artery stenosis, cervical plexus block, regional anesthesia, ultrasonography, facial nerve block

\section{Introduction}

Ultrasound-guided cervical plexus blocks (CPB) are established anesthetic procedures for carotid endarterectomies. The sensory branches of the cervical plexus (segments C2C4: greater auricular nerve, lesser occipital nerve, transverse cervical nerve [TCN], and supraclavicular nerves) emerge in the interfacial compartment (between the superficial and the prevertebral layers of the cervical fascia), piercing the superficial layer of the cervical fascia behind the sternocleidomastoid muscle (SCMM) at C4 or C5 transverse process. $^{1,2}$ They innervate the skin and fascia covering the auricle, mastoid process, anterolateral part of the neck, shoulder, clavicle, and superior aspect of the chest. ${ }^{2}$

However, CPB does not always produce complete anesthesia for carotid endarterectomy. Additional administration of opioids and/or local anesthetics is common
Correspondence: Ronald Seide

Asklepios Medical Center, Department of Anesthesiology and Intensive Care, Am

Klinikum I, Schwedt, 16303, Germany

Tel +49333253452l

Email ronald-seidel@t-online.de 
and has been reported previously. ${ }^{3-9}$ This required supplementation may result from incomplete CPB and also from additional innervation by cranial nerves (V, VII, IX, X, and $\mathrm{XI}$ ) and the sympathetic trunk (ST). ${ }^{7,10-12}$

Carotid endarterectomies typically involve painful surgical steps, including skin incision involving the dermatomes and the platysma (co-innervation by VII), vessel preparation (carotid plexus innervation: IX, X, ST), and potential retromandibular retractor insertion (co-innervation by $\mathrm{V}$, VII). ${ }^{7-9,13,14}$ The quality of anesthesia during skin incision is often decisive in regard to further patient comfort.

The TCN (sensory fibers) has caudal and cranial branches. The cranial branches form anastomoses (superficial cervical ansa [SCA]) with the facial nerve (cervical branch VII [CB VII], sensory and motor fibers) (Figures 1 and 2). ${ }^{1,10-12}$ From this loop, the platysma, skin, and fascia are innervated in the

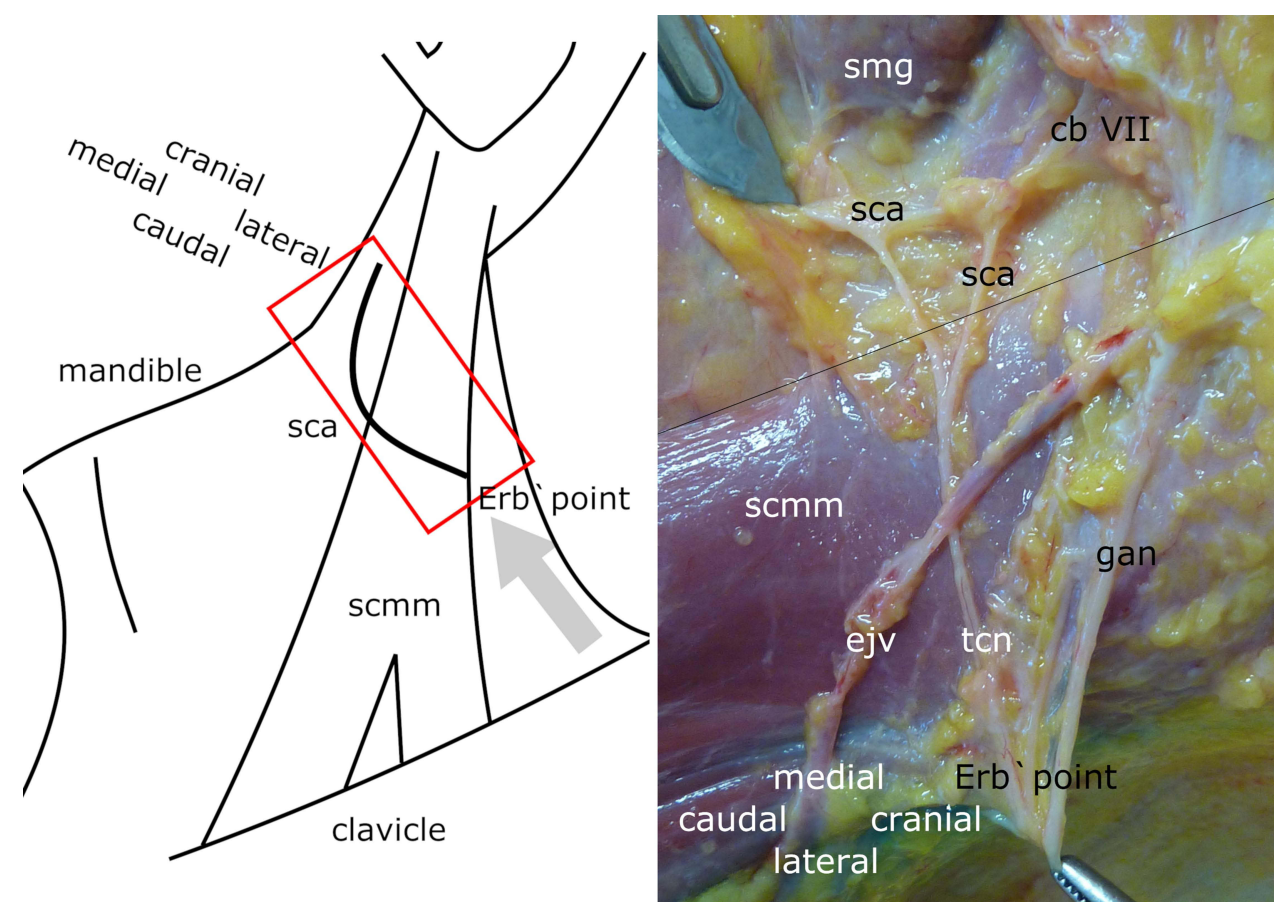

Figure I Superficial cervical ansa (Left Side). Left panel: Left side of neck (schematic): Red rectangle indicates image section in Figure I (right panel). Grey arrow indicates direction of view in Figure I (right panel). Right panel: Left side of neck (anatomical preparation): Skin and platysma are removed. ejv - external jugular vein, tcn - transverse cervical nerve (cranial branch), gan - greater auricular nerve (anterior and posterior branch), cb VII - cervical branch (facial nerve), sca - superficial cervical ansa, scmm sternocleidomastoid muscle, smg - submandibular gland, black line - anterior border of sternocleidomastoid muscle.

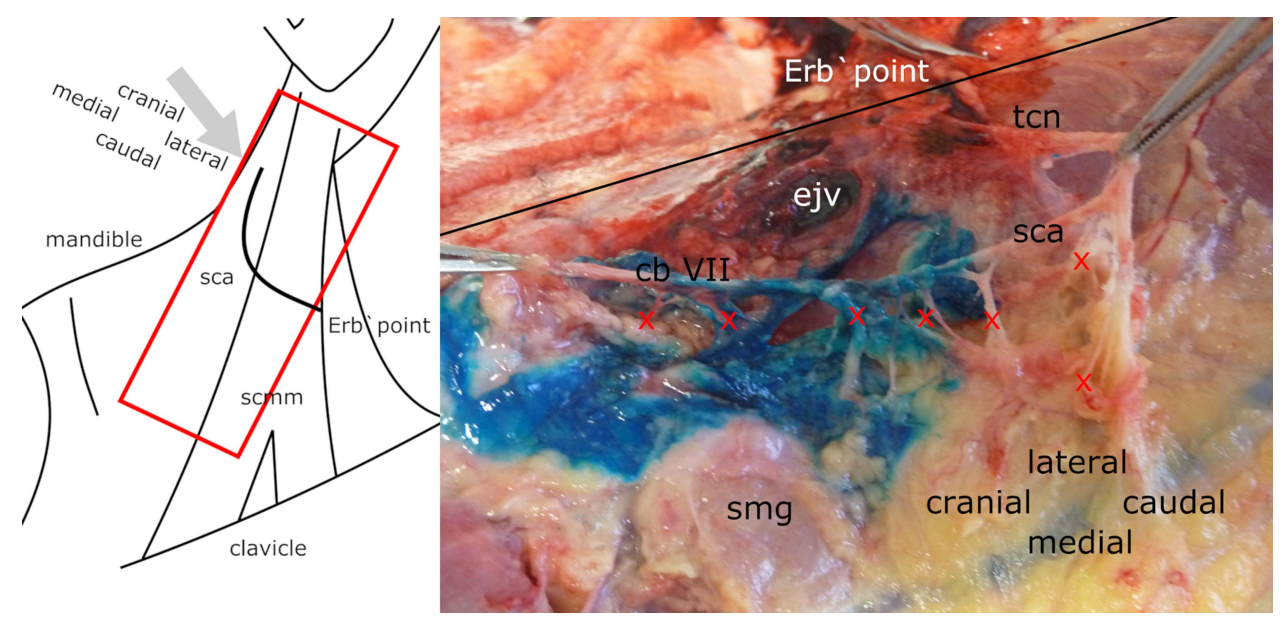

Figure 2 Superficial cervical ansa - dye staining and branches (Left Side). Left panel: Left side of neck (schematic): Red rectangle indicates image section in Figure 2 (right panel). Grey arrow indicates direction of view in Figure 2 (right panel). Right panel: Left side of neck (anatomical preparation): Skin and platysma are removed. tcn transverse cervical nerve, cb VII - cervical branch VII, sca - superficial cervical ansa, smg - submandibular gland, ejv - external jugular vein, red crosses - nerve branches that run to deep structures, possibly innervating the cervical fascia, black line - posterior border of sternocleidomastoid muscle. 
cranial section of the carotid triangle. ${ }^{13}$ The marginal mandibular branch VII (MMB VII) runs along the inferior edge of the mandible, innervating the lower lip muscles (Figure 3). ${ }^{1,14}$ These branches are partially cut during the initial skin incision. The quality of anesthesia in carotid endarterectomies could be potentially improved by blocking these nerves. Therefore, the present anatomical study aimed to develop a technique to block the SCA with defined sonoanatomic landmarks. Despite current discussions on fasciae nomenclature, the traditional classification technique was applied in the present study. ${ }^{15}$

\section{Materials and Methods}

The study was performed on eight (16 injections) unfixed donor cadavers (Caucasian; five males and four females; age: 66-90 years) who had donated their bodies for education or research purposes to the Institute of Anatomy, University of Rostock, Germany. Ethics was approved by the Ethics Committee (A 2016 0083) of the University of Rostock.

The cadavers were placed for puncture in a supine position with head slightly tilted to the opposite side. Injections $(5 \mathrm{~mL})$ of Alcian Blue 8GX dye (SigmaAldrich, St. Louis, MO, USA) were performed using a Sonoplex ${ }^{\circledR} 22$ G $50 \mathrm{~mm}$ cannula (Pajunk, Geisingen, Germany). The ultrasound device used was a Sonosite EDGE II $^{\circledR}$ with a $38 \mathrm{~mm}$ linear transducer $(6-13 \mathrm{MHz})$ (Sonosite, Bothell, WA, USA).

The subplatysmal SCA block targeted anastomoses between the TCN and branches of the facial nerve (CB VII, MMB VII) between the anterior margin of the SCMM and the submandibular gland (Figures 3 and 4; Supplementary video). First, the transducer was placed transversely at the level of the $\mathrm{C} 4$ transverse process. Next, the transducer was rotated slightly (medial end cranial) and positioned until contact was made with the mandible. Subsequently, the transducer was placed over the cranial aspect of the carotid triangle. The resulting ultrasound image depicted the following sonoanatomic landmarks: submandibular gland (medial, cranial) and anterior margin of the SCMM (lateral, caudal). The puncture was performed in-plane laterally/caudally.

Immediately following the injection, dye spread was assessed by anatomical preparation and photo documentation.

\section{Results}

Results of the anatomical preparations are summarized in Table 1. The SCA was identified and stained in all preparations (Figures 1-3). In 13 of 16 (81\%) cases, the cervical branch of the facial nerve could be visualized via ultrasound between the anterior margin of the SCMM and the submandibular gland. In 10 cases, the preparation was extended to the mandible to visualize the MMB VII, which was stained in all cases.

\section{Discussion \\ Injection Technique}

This anatomic case series demonstrated that subplatysmal infiltration with dye below the mandible results in staining of the cervical and marginal mandibular branches of the

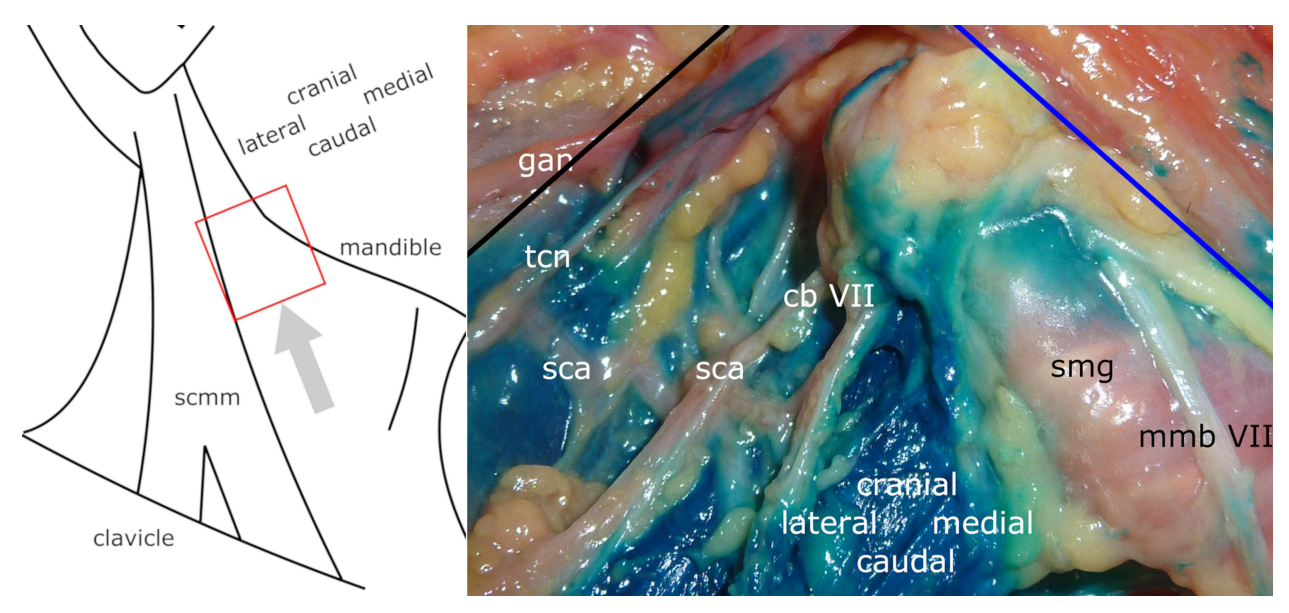

Figure 3 Superficial cervical ansa - dye staining and marginal mandibular branch VII (Right Side). Left panel: Right side of neck (schematic): Red rectangle indicates image section in right panel. Grey arrow indicates direction of view in right panel. Right panel: Right side of neck (anatomical preparation): Skin and platysma are removed. ten transverse cervical nerve, gan - greater auricular nerve, mmb VII - marginal mandibular branch (facial nerve), cb VII - cervical branch (facial nerve), sca - superficial cervical ansa, smg - submandibular gland, black line - anterior border of sternocleidomastoid muscle, blue line - inferior edge of mandible. 


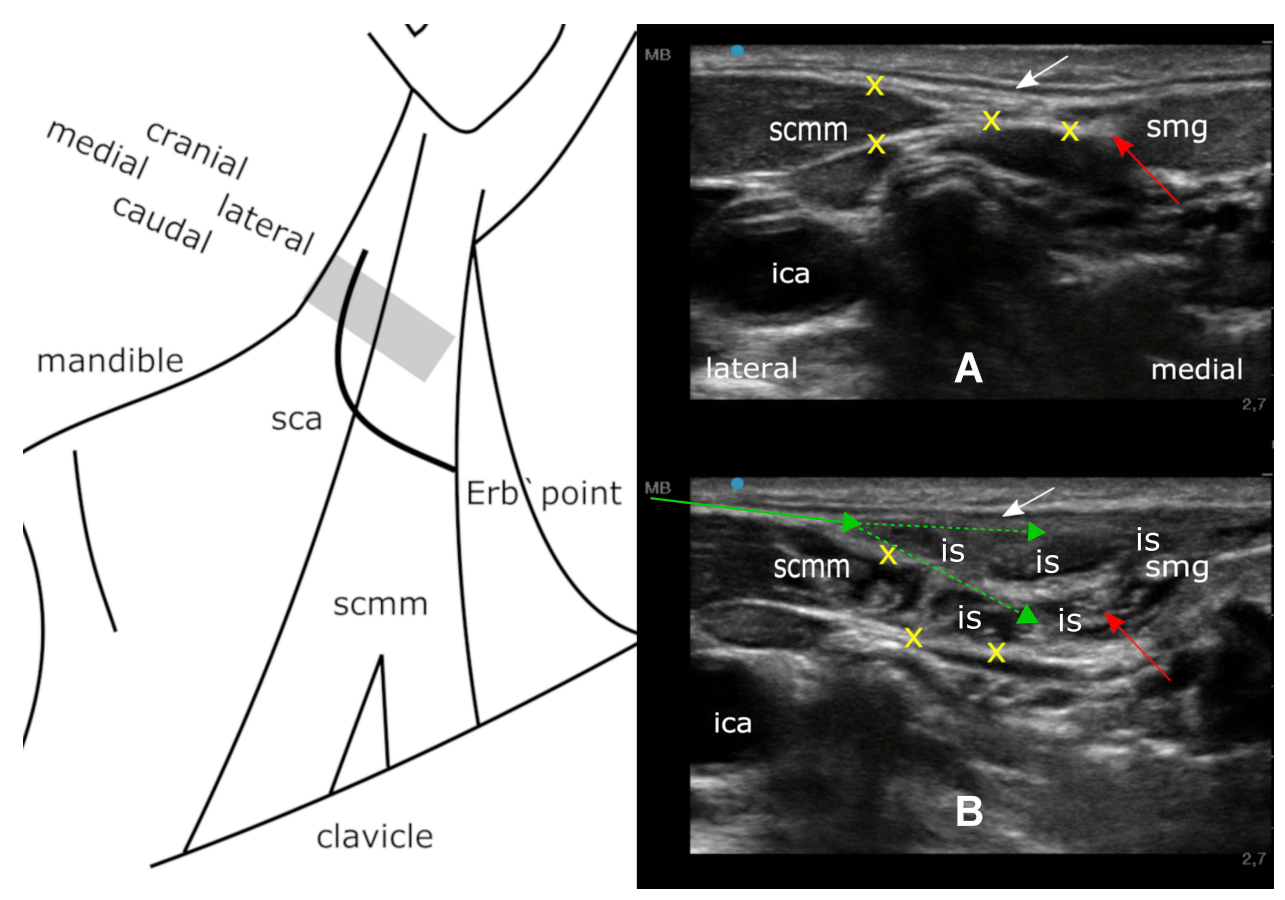

Figure 4 Regional anesthesia technique (Left Side). Left panel: Left side of neck (schematic): Grey rectangle indicates transducer position in right panel. Right panel: Block of the superficial cervical ansa (sonographic representation). White arrow - platysma, red arrow - cervical branch of the facial nerve (CB VII), green arrow - initial needle position, green dashed arrows - needle advance during injection, yellow crosses - superficial cervical fascia, smg - submandibular gland, scmm - sternocleidomastoid muscle, ica - internal carotid artery, is - injectate spread. A (top panel): before injection. B (bottom panel): after injection.

facial nerve. These branches anastomose with the TCN (cervical plexus), forming the SCA. The latter is potentially the anatomical basis for suboptimal anesthetic quality in the cranial section of the skin incision and during retromandibular retractor insertion. The former comprises various anatomical structures potentially with

Table I Results

\begin{tabular}{|c|c|c|c|c|}
\hline Preparation (Side) & Gender/Age (Years) & Staining: SCA & Staining: MMB VII & Sonographic Imaging: CB VII \\
\hline I (left) & \multirow[t]{2}{*}{ Male/77 } & Yes & Yes & Yes \\
\hline 2 (right) & & Yes & Yes & Yes \\
\hline 3 (left) & \multirow[t]{2}{*}{ Male/66 } & Yes & Not Dissected & Yes \\
\hline 4 (right) & & Yes & Not dissected & No \\
\hline 5 (left) & \multirow[t]{2}{*}{ Male/90 } & Yes & Not dissected & Yes \\
\hline 6 (right) & & Yes & Not dissected & Yes \\
\hline 7 (left) & \multirow[t]{2}{*}{ Male/7I } & Yes & Yes & Yes \\
\hline 8 (right) & & Yes & Yes & Yes \\
\hline 9 (left) & \multirow[t]{2}{*}{ Female/8I } & Yes & Yes & Yes \\
\hline 10 (right) & & Yes & Yes & Yes \\
\hline II (left) & \multirow[t]{2}{*}{ Female/87 } & Yes & Yes & Yes \\
\hline 12 (right) & & Yes & Yes & Yes \\
\hline 13 (left) & \multirow[t]{2}{*}{ Female/66 } & Yes & Not dissected & No \\
\hline I4 (right) & & Yes & Not dissected & No \\
\hline 15 (left) & \multirow[t]{2}{*}{ Female/88 } & Yes & Yes & Yes \\
\hline I6 (right) & & Yes & Yes & Yes \\
\hline
\end{tabular}


different innervations, including the skin (dermatomes), the platysma (myotomes), and the superficial layer of the cervical fascia (fasciotomes).

The sono-anatomic landmarks of the subplatysmal SCA block presented in the current study include the anterior border of the SCMM, submandibular gland, and platysma (Figure 4). Recently, this concept was tested in a prospective pilot study in a three-step injection technique (first injection: intermediate $\mathrm{CPB}$, perivascular infiltration, second injection: subplatysmal SCA block). ${ }^{16}$ In this study, block-related peripheral facial nerve palsy occurred in 24 of 28 cases, demonstrating evidence of MMB VII blockage. Thus, for routine use, the authors utilize a short-acting local anesthetic $(5 \mathrm{~mL}$ of $1 \%$ prilocaine) to avoid misinterpretation as a postoperative neurological deficit.

Essentially, the subplatysmal SCA block is a compartment block. In many cases (81\%), CB VII may be visualized before or during application of the local anesthetic (Figure 4, Supplementary Video and Table 1). It can be traced by ultrasound to the fascia of the submandibular gland at its inferior border. However, visualization is likely not required for a successful blockade. The goal is to achieve an even distribution of local anesthetic between the medial border of the sternocleidomastoid muscle and the submandibular gland.

Overall, this technique is a simple, superficial nerve block with clear sonoanatomic landmarks that could improve patient comfort in carotid endarterectomies.

\section{Alternative Injection Techniques}

The SCA may be anesthetized via subcutaneous infiltration within the carotid triangle. Interestingly, in addition to the CPB, this type of infiltration has been described by some authors as part of the primary block technique. ${ }^{3-5}$ However, these studies did not describe branches of the facial nerve or the SCA as targets for the local anesthetic injection. Rather, this approach was based on clinical experience in that $\mathrm{CPB}$ does not always result in complete anesthesia of the skin incision.

Pandit and colleagues performed a fan-shaped subcutaneous infiltration "toward the midline of the neck" from the posterior margin of the SCMM, in addition to the superficial $\mathrm{CPB}^{5}$ In another study, Calderon and coworkers combined intermediate $\mathrm{CPB}$ with subcutaneous infiltration of the incision line $(5-10 \mathrm{~mL}$ local anesthetic). ${ }^{3}$ And finally, Casutt et al omitted a CPB for carotid endarterectomies and only performed subcutaneous (incision line) and perivascular (carotid sheath) infiltration with $15 \mathrm{~mL}$ of local anesthetic each. ${ }^{4}$ However, the advantage of ultrasound guidance potentially lies in targeted subplatysmal (diffusion barrier) injection of small volumes $(5 \mathrm{~mL})$ of local anesthetic without vascular puncture.

\section{Study Limitations and Controversies}

The present study used a limited number of anatomical preparations. Furthermore, evidence of an improvement in the quality of anesthesia in randomized clinical trials is pending.

CB VII is described in the literature predominantly as a motor nerve innervating the platysma. ${ }^{13}$ Its importance for sensory innervation (skin, fascia) is not yet welldefined. To our knowledge, we are not aware of any anatomical studies on the fiber quality of the SCA. The existence of nociceptive fibers in fascial tissue and their significance is currently under investigation. ${ }^{17,18}$

The deep cervical ansa (current nomenclature: ansa cervicalis) is part of the cervical plexus (C1-C3) and provides motor innervation to the infrahyoid muscles. It is located below the SCMM, crosses the internal jugular vein, finally attaching to the hypoglossal nerve. In contrast, the SCA lies subplatysmal in the carotid triangle and is formed by anastomoses between the cervical plexus and CB VII. ${ }^{1}$

\section{Conclusion}

This anatomical study demonstrates an ultrasound-guided block of anastomoses between the transverse cervical nerve and the cervical branch VII (subplatysmal SCA block) to improve the quality of anesthesia for carotid endarterectomies.

Further studies on the fiber quality of the involved nerves as well as the fascia innervation are necessary. The anatomical data and conclusions presented need to be verified in randomized clinical trials.

\section{Abbreviations}

CB VII, cervical branch VII; CPB, cervical plexus block; MMB VII, marginal mandibular branch VII; SCA, superficial cervical ansa; SCMM, sternocleidomastoid muscle; ST, sympathetic trunk; TCN, transverse cervical nerve; V, trigeminal nerve; VII, facial nerve; IX, glossopharyngeal nerve; $\mathrm{X}$, vagal nerve; $\mathrm{XI}$, spinal accessory nerve. 


\section{Acknowledgments}

Editorial assistance, in the form of language editing and correction, was provided by XpertScientific Editing and Consulting Services.

\section{Disclosure}

The authors report no conflicts of interest in this work.

\section{References}

1. Lanz T, Von, Wachsmuth W. Hals. In: Lanz T, Von, Wachsmuth W, editors. Praktische Anatomie. Ein Lehr- und Hilfsbuch der anatomischen Grundlagen ärztlichen Handelns. Berlin: Springer; 2003:37-62.

2. Greengrass RA, Narouze S, Bendtsen TF, et al. Cervical plexus and greater occipital nerve blocks: controversies and technique update. Reg Anesth Pain Med. 2019;4:623-626. doi:10.1136/rapm-2018100261

3. Calderon AL, Zetlaoui P, Benatir F, et al. Ultrasound-guided intermediate cervical plexus block for carotid endarterectomy using a new anterior approach: a two-centre prospective observational study. Anaesthesia. 2015;70:445-451. doi:10.1111/anae.12960

4. Casutt M, Breitenmoser I, Lennart W, et al. Ultrasound-guided carotid sheath block for carotid endarterectomy: a case series of the spread of injectate. Heart Lung Vessels. 2015;7:168-176.

5. Pandit JJ, Bree S, Dillon P, et al. A comparison of superficial versus combined (superficial and deep) cervical plexus block for carotid endarterectomy: a prospective, randomized study. Anesth Analg. 2000;91:781-786. doi:10.1097/00000539-200010000-00004

6. Martusevicius R, Swiatek F, Joergensen LG, et al. Ultrasound-guided locoregional anaesthesia for carotid endarterectomy: a prospective observational study. Eur J Vasc Endovasc Surg. 2012;44:27-30. doi:10.1016/j.ejvs.2012.04.008

7. Ozturk NK, Kavakli AS, Sagdic K, et al. A randomized controlled trial examining the effect of the addition of the mandibular block to cervical plexus block for carotid endarterectomy. $J$ Cardiothorac Vasc Anesth. 2018;32:877-882. doi:10.1053/j.jvca.2017.06.034
8. Ramachandran SK, Picton P, Shanks A, et al. Comparison of intermediate vs subcutaneous cervical plexus block for carotid endarterectomy. $\mathrm{Br}$ J Anaesth. 2011;107:157-163. doi:10.1093/bja/aer118

9. Seidel R, Zukowski K, Wree A, et al. Ultrasound-guided intermediate cervical plexus block and perivascular local anesthetic infiltration for carotid endarterectomy: a randomized controlled trial. Anaesthesist. 2016;65:917-924. doi:10.1007/s00101-016-0230-z

10. Ella B, Langbour N, Caix P, et al. Transverse cervical and great auricular nerve distribution in the mandibular area: a study in human cadavers. Clin Anat. 2015;8:109-117.

11. Shoja MM, Oyesiku NM, Griessenauer CJ, et al. Anastomoses between lower cranial and upper cervical nerves: a comprehensive review with potential significance during skull base and neck operations, part I: trigeminal, facial and vestibulocochlear nerves. Clin Anat. 2014;27:118-130. doi:10.1002/ca.22340

12. Shoja MM, Oyesiku NM, Shokouhi G, et al. Anastomoses between lower cranial and upper cervical nerves: a comprehensive review with potential significance during skull base and neck operations, part II: glossopharyngeal, vagus, accessory, hypoglossal and cervical spinal nerves 1-4. Clin Anat. 2014;27:131-144. doi:10.1002/ca.22342

13. Hwang K, Kim JY, Lim JH. Anatomy of the platysma muscle. J Craniofac Surg. 2017;28:539-542. doi:10.1097/SCS.0000000000003318

14. Marcuzzo AV, Šuran-brunelli AN, Dal Cin E, et al. Surgical anatomy of the marginal mandibular nerve: a systematic review and meta-analysis. Clin Anat. 2020;33:739-750. doi:10.1002/ca.23497

15. Adstrum S, Hedley G, Schleip R, et al. Defining the fascial system. J Bodyw Mov Ther. 2017;21(1):173-177. doi:10.1016/j.jbmt.2016.11.003

16. Seidel R, Zukowski K, Wree A, et al. Ultrasound-guided intermediate cervical plexus and additional peripheral facial nerve block for carotid endarterectomy: a prospective pilot study. Anaesthesist. 2018;67:907-913. doi:10.1007/s00101-018-0493-7

17. Mense S. Innervation of the thoracolumbar fascia. Eur J Trans Myol. 2019;29:151-158. doi:10.4081/ejtm.2019.8297

18. Stecco C, Pirri C, Fede C, et al. Dermatome and Fasciotome. Clin Anat. 2019;32:896-902. doi:10.1002/ca.23408
Local and Regional Anesthesia

\section{Publish your work in this journal}

Local and Regional Anesthesia is an international, peer-reviewed, open access journal publishing on the development, pharmacology, delivery and targeting and clinical use of local and regional anesthetics and analgesics. The journal welcomes submitted papers covering original research, basic science, clinical studies, reviews \& evaluations, guidelines, expert opinion and commentary, case reports and extended reports. The manuscript management system is completely online and includes a very quick and fair peer-review system, which is all easy to use. Visit http://www.dovepress.com/testimonials. php to read real quotes from published authors. 